\title{
Toughening mechanisms for the attachment of architectured materials: The mechanics of the tendon enthesis
}

\section{Short title: Toughening mechanisms at the tendon enthesis}

\section{Authors}

Mikhail Golman ${ }^{1,2}$, Adam C. Abraham ${ }^{1}$, Iden Kurtaliaj ${ }^{1,2}$, Brittany P. Marshall ${ }^{1,2}$, Yizhong Jenny $\mathrm{Hu}^{2}$, Andrea G. Schwartz ${ }^{3}$, X. Edward Guo ${ }^{2}$, Victor Birman ${ }^{4}$, Philipp J. Thurner ${ }^{5}$, Guy M. Genin $^{3, *}$, Stavros Thomopoulos ${ }^{1,2, *}$

\section{Affiliations}

1 - Department of Orthopedic Surgery, Columbia University, New York, NY

2 - Department of Biomedical Engineering, Columbia University, New York, NY

3 - NSF Science and Technology Center for Engineering Mechanobiology, Washington University, St. Louis, MO

4 - Missouri University of Science and Technology, Rolla, MO

5 - Institute of Lightweight Design and Structural Biomechanics, Vienna University of

Technology, Vienna, Austria

\section{* Corresponding Authors:}

Stavros Thomopoulos, $\mathrm{PhD}$

Columbia University

Black Building, Room 1408

$650 \mathrm{~W} 168 \mathrm{ST}$

New York, NY 10032-3702

Phone: 212-305-5124

Email:sat2@columbia.edu

Guy M. Genin, $\mathrm{PhD}$

Washington University

1 Brookings Drive

3 St. Louis, MO 63130

4 Phone: 314-935-5660

5 Email: genin@wustl.edu 


\section{$37 \quad$ Abstract}

38 Architectured materials offer tailored mechanical properties but are limited in engineering 39 applications due to challenges in maintaining toughness across their attachments. The enthesis 40 connects tendon and bone, two vastly different architectured materials, and exhibits toughness 41 across a wide range of loadings. Understanding the mechanisms by which this is achieved could 42 inform the development of engineered attachments. Integrating experiments, simulations, and 43 novel imaging that enabled simultaneous observation of mineralized and unmineralized tissues, 44 we identified putative mechanisms of enthesis toughening in a mouse model and then 45 manipulated these mechanisms via in vivo control of mineralization and architecture. Imaging 46 uncovered a fibrous architecture within the enthesis that controls trade-offs between strength and 47 toughness. In vivo models of pathology revealed architectural adaptations that optimize these 48 trade-offs through cross-scale mechanisms including nanoscale protein denaturation, milliscale 49 load-sharing, and macroscale energy absorption. Results suggest strategies for optimizing 50 architecture for tough bimaterial attachments in medicine and engineering.

53 The architecture of the tendon-to-bone attachment is designed for toughness. 


\section{Introduction}

Materials whose micro- and meso-scale architectures endow them with useful mechanical functions are found throughout nature and, more recently, in engineering (1-4). However, engineering application of such architectured materials is limited by the challenge of attaching them (5). Typical features of architectured materials (e.g., microtruss composites) lead to local elevations in stress that can reduce strength (i.e., stress required to break the material) and toughness (i.e., the energy absorbed prior to breaking the material) when they are connected to other materials $(6,7)$. Natural materials provide a rich source of inspiration for the design and attachment of architectured engineering materials. For example, the tendon enthesis illustrates a number of novel and often counter-intuitive ways by which architectured materials can be effectively connected. Tendon and bone, tissues with a two orders-of-magnitude difference in modulus, display a hierarchical architecture ranging from nanometer-scale triple-helix tropocollagen molecules to sub-micrometer-diameter fibrils to 10-100 micrometer-diameter fibers that extend over millimeters (8). Across species, strong attachment of tendon and bone arises from a zone of compliant transitional tissue $(9,10)$ that mitigates stress concentrations through allometric scaling of geometry (11) and through functional gradations of both fiber orientation $(12,13)$ and bioapatite mineral $(14-17)$. These aspects of enthesis architecture are not recreated following injury, and surgical repairs thus often fail $(18,19)$. Despite progress in understanding how the enthesis achieves a strong attachment under sub-damage loading regimes, it remains unclear how toughness is achieved to prevent interfacial failure. Understanding these mechanisms will guide engineering and medical approaches to bimaterial attachment.

We therefore aimed to identify enthesis architectural and compositional toughening mechanisms in mice using imaging, biomechanical testing, and mathematical modeling. A novel micro computed tomography (microCT) technique was developed to simultaneously visualize the mineralized and unmineralized fibrous networks within the tendon-bone attachment at sub- 
micrometer resolution. We manipulated the fibrous network through pathophysiologic loading in vivo in a mouse model and quantified how monotonic (acute) and cyclical (degenerative) loading affected enthesis strength and toughness. Biomechanical analysis and numerical simulation supported our hypothesis that architectural toughening arises from the composition (nanoscale mineral and proteoglycans), structure (microscale collagen organization and recruitment), and position (macroscale loading angle) of the transitional material. Physiologically, enthesis composition and micro-structure in vivo adapted to loading in a way that revealed a trade-off between strength and toughness. These features of the adaptable, architectured, fibrous enthesis have direct implications for tough attachment between dissimilar materials, facilitating improved design of surgical and tissue engineered solutions for tendon-to-bone repair.

\section{Results}

\section{Attachment at the enthesis relies on a fibrous architectured material system}

Using microCT imaging with mercury (II) chloride staining, we obtained simultaneous, sub-micrometer imaging of unmineralized and mineralized tissue in the mouse supraspinatus tendon enthesis (Fig. 1A) and discovered that the function of the enthesis had been previously misunderstood. Hidden within the well-known attachment footprint (11) (Fig. 1B within blue dotted line) was a smaller, denser "primary" insertion site where tendon fibers directly inserted into bone over $30 \% \pm 3.5 \%$ of the footprint area (Fig. 1C, within green dotted line, FigS1, Movie S1). Collagen fibers were continuous from muscle to bone but branched into smaller diameter fibers as they inserted into bone on one end, as previously described $(9,20)$, or muscle at the other.

To test the hypothesis that the primary insertion site was responsible for load transfer, we stretched supraspinatus tendon enthesis specimens to failure quasi-statically. The enthesis failed through avulsion of a bone plug (Fig. 1C) over $22.4 \% \pm 6.2 \%\left(0.31 \pm 0.09 \mathrm{~mm}^{2}\right)$ of the apparent 
54 insertion site (Fig.S1C), with the majority of the primary insertion avulsed, but with peritenon

55 tissue surrounding the primary insertion site still attached (Fig. S2A, Movie S2). Failure occurred

J6 catastrophically, with little resistance to post-failure force (Fig. S2B), supporting our hypothesis.

We next asked how the primary insertion resisted failure loads. Although failure was expected at the mineralized interface within fibrocartilage where the stress concentrations were predicted to occur $(21,22)$, this was not observed, indicating mechanisms to alleviate these stress concentrations. Failure occurred either at the interface between mineralized fibrocartilage and bone (MF-B failure type), or within trabecular bone (B-T failure type) (Fig. 1 C - I; Fig. S3A, Movie S3) and in all cases with crack propagation around the avulsion site (scanning electron microscopy, Fig. 1J and Fig. 3SB). For this loading, the fibrous primary enthesis was thus tougher than cortical bone, with the more compliant fibrocartilage storing enough energy to fracture and avulse bone.

\section{Multiscale toughening mechanisms enable resistance to cyclical loading}

The enthesis is durable against the complex and repeated loadings of daily activities (23), but failure mechanisms change with loading regime and age. Avulsions are common in high-impact injuries for pediatric patients (24), but rupture at the tendon end of enthesis is prominent in degenerated rotator cuffs of adult patients (25-27). We therefore hypothesized that toughening mechanisms depend upon the loading regime.

In response to acute loading (monotonic tension across a range of loading rates) or fatigue loading (cyclic loading at $2 \mathrm{~Hz}$, either $1-20 \%$ or $20-70 \%$ failure load), three distinct failure modes were observed (Fig. 2 A-E): bone avulsion, tendon mid-substance failure, and tendon-bone interface failure. Acute loading led primarily to avulsion, regardless of loading rate. Although 
27 enthesis mechanical properties were largely strain-rate insensitive, like tendon properties $(28,29)$,

28 strength (failure load) and toughness (work to failure, calculated as the area under the force-

29 displacement curve) increased at higher strain rates by as much as 1.4-fold $(p<0.0001)$ and 1.6-

30 fold $(p<0.01)$, respectively, compared to that of control test case ( $n=10-12 /$ case, Fig. 2 C and D;

31 Fig.S4 A-C). Notably, the area and number of fragments of the avulsed region increased with

32 loading rate (Fig. S4 C-F). In contrast to acute loading, all cyclically loaded samples (High: $2 \mathrm{~Hz}$,

$3320-70 \%$ of strength) failed in the unmineralized fibrocartilage portion of the attachment

34 (“insertion failure", Fig 2E). Samples cyclically loaded at lower, physiologically relevant loads

35 (Low: 2Hz, 1\%-20\% of strength), did not fail, even after 100,000 cycles (Fig. 2E), indicating that

36 these loading levels were under the enthesis fatigue limit. Results thus suggested that the

37 mechanisms protecting fibrocartilaginous enthesis tissue might be gradually attenuated under

38 sufficiently severe cyclical loading.

quantified molecular damage under the various loading regimes using fluorescein-labeled

41 collagen hybridizing peptide (F-CHP) $(30,31)$. Whole-sample imaging of F-CHP fluorescence

42 intensity, indicative of collagen damage, increased with applied load or number of cycles (Fig.

$432 \mathrm{~F}$, top). In monotonic loading, fluorescent signal accrued near the primary insertion site when

44 loads exceeded 3 N. Under cyclic loading, signal was concentrated in a few fibers near the tendon

45 mid-substance between 10,000 and 40,000 cycles, then propagated down the entire tendon in

46 concentrated bands (Fig. 2F, bottom). This revealed that, in monotonic loading to failure, energy

47 sufficient to avulse bone was stored in the enthesis with relatively little energy dissipation, while

48 in cyclical loading, energy was absorbed by damage within the tendon and enthesis, eventually

49 leading to failure within the unmineralized tissues. Thus, the enthesis contains fiber-level

50 toughening mechanisms to resist monotonic loading and an underlying nanoscale mechanism to

51 resist cyclical loading. 
Differential recruitment of collagen fibers enables toughness across loading directions

Based upon observations of the fibrous character to the enthesis, we hypothesized that these nanoscale mechanisms are supplemented by macroscale toughening mechanisms to resist failure across a range of directions (i.e., shoulder abduction angle, Fig. 3A-B). Enthesis behavior, including strength and stiffness, varied with the angle of abduction (Fig. 3C-F). This was a surprise given the shoulder's ability to resist injury across its broad range of motion (32). $\mathrm{HgCl}_{2}$ enhanced microCT images revealed that fibers engaged or buckled depending upon loading (Fig. 3B top, Fig.S5A), consistent with fiber recruitment models of tendon mechanics (33) and rotator cuff injury (26). We therefore developed a series of experiments and models to determine how abduction-dependent fiber architecture and recruitment dictated enthesis mechanics.

Imaging at $5 \mu \mathrm{m}$ resolution revealed that the collagen fibers of the supraspinatus tendon enthesis engaged at low abduction angles $\left(0^{\circ}\right.$ and $\left.30^{\circ}\right)$ and buckled at high abduction angles $\left(90^{\circ}\right.$ and $120^{\circ}$ ) (Fig. 3B, top row outlined in blue, Video S4). Furthermore, imaging at $0.75 \mu \mathrm{m}$ resolution confirmed that outer (bursal-side) fibers were longer than inner (articular-side) fibers (Figure 3B, bottom row and Fig.S5A), as similar to what previously described in human $(34,35)$. This indicated that both inner and outer fibers engaged to carry loads at low angles of abduction, but only inner fibers engaged at high angles, with outer fibers remaining slack.

We then explored whether these changes in microscale fiber engagement with shoulder abduction could explain the observed macroscale adaptations in tendon enthesis toughness and strength using a numerical model (Supplemental Text). The model idealized the geometry of the humeral head as a circular bone ridge beneath linear elastic fibers of pre-defined thickness and spacing. Fibers engaged, re-oriented, and contacted neighboring fibers or the humeral head during 
75 loading (Fig. 3A bottom, Fig. 5SB) in a way that varied with abduction, and that reproduced

76 trends observed in our experiments (Fig. 3G and H): normalized strength and toughness increased

77 with decreasing abduction angle, while stiffness decreased with decreasing abduction angle.

78 These results thus supported the hypothesis that abduction-dependent fiber recruitment was a

79 factor in failure patterns, with the displacement needed to engage (recruit) all fibers lowest at $60^{\circ}$

80 of abduction (Fig. 3I), and four times higher at $120^{\circ}$ than at $90^{\circ}$ of abduction. When considering

31 failure behavior across the physiological range of shoulder abduction (Fig. 3J), strength decreased

82 with abduction angle from $90^{\circ}$ to $0^{\circ}$, while toughness increased; strength and toughness decreased

83 dramatically beyond $90^{\circ}$ of abduction.

From the perspective of shoulder physiology, results inform our understanding of rotator

The fibrous architecture of the tendon enthesis enabled its fibers to reorient, recruit, and subsequently rupture to balance strength and toughness across a wide range of motion, a tradeoff well known in material design (7). The healthy enthesis appeared optimized for toughness, with gains in toughness associated with changing abduction angle achieved through comparably modest losses in strength (Fig 3J). This is somewhat analogous to brittle matrix fibrous composites achieving toughness at the expense of strength $(17,37)$, and how microscale interdigitation of the tendon enthesis toughens attachments (15). The trade-off was particularly apparent at lower abduction angles, where rotator cuff muscles were most engaged and enthesis 
99 loads were highest (38). Although factors such as viscoelasticity and post-yield behavior also

j0 contribute to enthesis toughness, the current modeling and experimental results support a clear

01 role for abduction position-dependent kinematics driving tendon enthesis toughness in the rotator

32 cuff.

34 Tendon enthesis strength is determined by mineral composition

A spatial gradient in mineral stiffens the enthesis, especially beyond a percolation threshold (39), and mitigates stress concentrations (40). Proteoglycans stiffen and provide energy dissipation in articular cartilage (41). To test the hypothesis that these extracellular matrix components also contribute to enthesis toughness, each was chemically removed from the enthesis prior to mechanical testing (Fig. 4A, Fig.S7). We hypothesized that removal of mineral would reduce stiffness and strength, and that removal of proteoglycans would reduce toughness.

\section{Removal of mineral or proteoglycan did not significantly alter failure modes under} monotonic loading; samples failed primarily via bone avulsion, with 20\% (2/10 samples) of mineral depleted samples failing at the insertion (Fig. 4B, Fig. S8A and B). As hypothesized, removal of mineral decreased strength and stiffness ( $p<0.0001$, Fig. 4D and Fig.S8C), but also decreased toughness $(\mathrm{p}<0.0001$, Fig. $4 \mathrm{E})$. Contrary to the hypothesis, removal of proteoglycans did not change toughness, although decreases in strength and stiffness were observed, which were in agreement with prior findings at the scale of collagen fibrils (42). Of note, the proteoglycan depletion protocol used here removed proteoglycan in the unmineralized portion of the enthesis only (Fig. S7B), and therefore proteoglycan-mineral interactions cannot be ruled out.

Nevertheless, results demonstrate that mineral content is crucial for enthesis strength and toughness. 
The tendon enthesis actively adapts its architecture in vivo by controlling mineral composition and microarchitecture

It is well known that bone $(43,44)$ and entheses $(45)$ respond to loading by adapting their mineral content. To further elucidate how composition and architecture are modulated at the enthesis in vivo to produce toughness, we varied the loading environment of mouse shoulders via hypothesized that modifications to in vivo loading would lead to architectural adaptations that control strength and toughness.

Regardless of treatment, all specimens failed via avulsion under monotonic loading (Fig. 5B). Healthy and overuse-degenerated attachments failed catastrophically, showing little postyield behavior, while underuse-degenerated attachments failed at lower forces and showed distinct post-yield behavior (Fig. 5C). Pathologic loading led to distinct changes to enthesis failure pattern. Underuse increased fracture area by as much as 1.9-fold compared to that of control ( $<<0.01$, Fig. 5D). While overuse-degenerated entheses failed primarily with one bony avulsed fragment, failures in underuse-degenerated attachments showed multiple fragments of avulsed bone (Fig. 5E). Overuse and underuse led to a shift in the fracture location: overuse resulted in more failures at the MF-B interface while underuse resulted in more failures at the B-T interface (Fig. 5F). Both overuse and underuse reduced toughness, but via different mechanisms. Overuse did not affect tendon enthesis strength (Fig. 5G) but led to an increase in stiffness ( $p<0.01$, Fig. $5 \mathrm{H})$, resulting in $\sim 30 \%$ decrease in toughness $(p<0.05$, Fig.5I). In contrast, underuse led to a decrease in strength $(p<0.01$, Fig. $5 \mathrm{G})$ and a decrease in stiffness $(p<0.05$, Fig $5 H)$, resulting in a decrease in toughness $(\mathrm{p}=0.08$, Fig. 5I). Hence, Loss in toughness in overuse 5 entheses was associated with reduced displacement at failure, without a change in strength; loss in 
toughness in underuse entheses was associated with reduced strength at failure, without a change in failure displacement.

To investigate the architectural adaptations underlying these effects, we characterized changes in the bone underlying the tendon enthesis. Bone morphometric analysis revealed that overuse led to up to $9 \%$ gain of bone volume in the humeral head (BV/TV, $<<0.01$, Fig. $5 \mathrm{~K})$, while underuse led to up to $24 \%$ loss in bone volume in the humeral head (BV/TV, $<<0.0001$, Fig. $5 \mathrm{~K}$ ) and up to $22 \%$ loss of bone mineral density underlying the attachment $(\mathrm{BMD}, \mathrm{p}<0.0001$, Fig. 5L). Study of individual trabecula, via three-dimensional segmentation of the trabecular network into rods and plate microarchitectures (46), showed that overuse increased the volume of load-bearing trabecular plates (pBV/TV) as much as $22 \%(\mathrm{p}<0.0001)$, while underuse decreases this as much as $30 \%(p<0.0001)$ (Fig. 5M). Overuse increased the thickness $(p<0.01)$ of individual trabeculae $30 \%$, while underuse decreased the number of trabecular plates by $15 \%$ $(\mathrm{p}<0.0001$, Fig. S10). The trabecular network of healthy, cage-active control samples had the highest density of trabecular plates oriented at $90^{\circ}-60^{\circ}$ relative to the dominant fiber direction in the supraspinatus tendon, and the lowest density of trabecular rods oriented in this range (Fig. $5 \mathrm{~N}$, Fig. S11). With overloading, trabecular plate density increased in $60^{\circ}-30^{\circ}(\mathrm{p}<0.05)$ and $30^{\circ}-0^{\circ}$ $(p<0.01)$ ranges, and with underuse, trabecular plate and rod loss occurred uniformly across all directions $(\mathrm{p}<0.05)$. These results demonstrate that overuse loading prompted active reinforcement whereas underloading prompted active removal of the trabecular architecture underneath the enthesis. Thus, the architecture of the bony structure at the tendon enthesis oriented to support and share the load into orientations of relatively low enthesis strength and toughness.

To understand which architectural features drove enthesis mechanical behavior, we correlated enthesis failure properties to bone and tendon microarchitecture using Pearson 
70 correlation (Fig. $5 \mathrm{O}$ and Fig. S12). Enthesis strength correlated strongly with BMD (R=0.60,

$71 \mathrm{p}<0.001)$, cortical thickness $(\mathrm{R}=0.69, \mathrm{p}<0.001)$, and trabecular plate thickness $(\mathrm{R}=0.59, \mathrm{p}<0.001)$,

72 but not with tendon cross-sectional area. Enthesis toughness correlated strongly with tendon

73 cross-sectional area $(\mathrm{R}=0.43, \mathrm{p}<0.05)$, and trended with mineralized fibrocartilage volume

$74(\mathrm{R}=0.30, \mathrm{p}=0.11)$. These results are consistent with clinical findings that the loss of mineralized

75 tissue at the attachment site correlates with higher rates of re-tearing following surgical repair 76 (47).

\section{Discussion}

This study revealed architectural toughening mechanisms at the enthesis, providing guidance for attachment of dissimilar materials (Fig. 6). First, energy storage in a compliant region of the fibrous attachment was protective, precluding fracture of the intricately architectured transitional tissue and instead leading to fracture of more easily regenerated bone. While counterintuitive, a tough, architectured compliant material attaching two dissimilar materials occurs across nature, e.g., in nacre (48), tooth enamel (49), and some mollusks (50). Compliant attachment layers in engineering have also been used in bottom-up and top-down fabrication of architecture materials (6), such as PMMAs inserted in between alumina layers (51), to absorb energy and channel crack propagation, and polymeric foams inserted into metallic foams (52). Second, the tendon enthesis harnesses its fibrous nature for effective load transfer. Nanoscale energy absorption by collagen molecules resists fatigue loading, while milliscale network behavior enables fiber reorientation, recruitment, and load sharing for toughness across loading directions. A similar concept has been applied to topologically interlocked material panels, with failure shared across contiguous panels and localized to repairable regions $(53,54)$. Distributions of fibers are further optimized at the enthesis to harness the toughness of the entire 
94 fibrous network at all loading directions, and to provide enhanced stiffness in the loading conditions for which muscle forces are highest. This relatively simple mechanism provides a principle that can be readily harnessed for engineering.

Additional features of the enthesis that will be more difficult to harness in engineering are compositional adaptions of architecture to physiologic loading. In vivo loading models revealed bony architecture actively remodeling to maintain strength along the axis of loading, while compromising overall toughness. Microstructural heterogeneity that toughens fibrous interfaces $(8,37)$ derives in part from mineral nanocrystal reorganization and reorientation $(16)$ but controlling these factors, as well as potential mineral binding proteins such as proteoglycans (55) and osteopontin (56), is currently beyond the scope of current top-down and bottom-up manufacturing techniques. Our findings demonstrated how the tendon enthesis achieves a remarkable balance between strength and toughness through its architecture to resist injurious loads. The toughening mechanisms identified here for the tendon enthesis provide guidance for improving enthesis surgical repair and enthesis tissue engineered scaffolds, as well as approaches for attachment of architectured engineering material systems.

\section{Materials and Methods}

\section{Sample preparation and study workflow}

All animal procedures were approved by the Columbia University Institutional Animal Care and

Use Committee. Supraspinatus tendon-to-bone attachment units (humerus-supraspintatus tendon-

4 supraspinatus muscle) were harvested from adult ( $>12$ weeks) male C57BL6/J mice $(n=275)$.

5 After dissection, samples were fresh-frozen in PBS and stored at $-20^{\circ} \mathrm{C}$. The experimental workflow was dependent on two categories: (1) unloaded/intact sample characterization (2) loaded sample characterization. For unloaded-sample characterization, defrosted samples were subjected to initial experimental protocol described in the sections below (i.e., secured at appropriate angle of abduction or chemically digested) and imaged via contrast enhanced 
microCT or via light microscopy, as the imaging techniques were terminal. For characterizing

21 samples undergoing loading, defrosted samples were first scanned by conventional microCT

22 before subjected to experimental protocol and mechanical testing. After mechanical testing, samples were secured at terminal displacements and either submerged in a 5\% mercury chloride ( $\mathrm{HgCl}_{2}$, Sigma-Aldrich) or fixed with $4 \%$ paraformaldehyde (Sigma-Aldrich) to analyze for macroscopic and fiber network level damage or molecular level (collagen) damage.

\section{Mechanical testing}

All samples were mechanically tested in a saline bath at $25^{\circ} \mathrm{C}$ to prevent thermal collagen denaturation on a table-top tensile tester (Electroforce 3230, TA Instruments) fitted with $10 \mathrm{lb.}$ load cell (TA instruments). Before testing, the supraspinatus muscle was carefully removed from supraspinatus-humerus unit. Samples were placed into custom 3D-printed fixtures (57) and supraspinatus tendon were secured between two layers of thin paper (Kimwipe) with a drop of cyanoacrylate adhesive (Loctite, Ultra Gel Control) before mounting onto custom grips. Samples were secured in fixtures and tested in an orientation corresponding to $90^{\circ}$ shoulder abduction unless otherwise noted. Specifically, to identify positional contributions to enthesis toughness, samples were fitted to 3D-printed fixtures that secured samples in an orientation corresponding to various angles of abductions $\left(0^{\circ}, 30^{\circ}, 60^{\circ}, 120^{\circ}, \mathrm{n}=10\right.$ per angle $)$. For all mechanical testing protocols, samples were first pre-loaded to $0.05 \mathrm{~N}$, pre-conditioned by applying 5 cycles of sinusoidal wave consisting of $5 \%$ strain and $0.2 \% / \mathrm{s}$, and rested for 300 seconds. The unloaded control group consisted of samples that were prepared and mounted in the mechanical tester, but not loaded $(\mathrm{n}=5)$.

Quasi-static and monotonic uniaxial loading: post pre-loading, pre-conditioning, and rest, samples were strained in tension at $0.2 \% / \mathrm{s}$ to failure (for all loading conditions unless specified otherwise below). The healthy failed control samples (CTRL) were healthy adult enthesis samples 
45 strained in tension at $0.2 \% / \mathrm{s}$ to failure in an orientation corresponding to $90^{\circ}$ abduction. For the

46 interrupted testing, samples were strained in tension at $0.2 \% / \mathrm{s}$ to $1 \mathrm{~N}, 2 \mathrm{~N}, 3 \mathrm{~N}$ ( $\mathrm{n}=3$ per rate). To

47 examine the role of strain rate in enthesis failure, samples were tested under three additional strain

48 rates $(2 \% / \mathrm{sec}, 20 \% / \mathrm{sec}, 200 \% / \mathrm{sec}, \mathrm{n}=10$ per rate $)$ until failure. Fatigue loading: after pre-

49 loading and preconditioning, samples were either subjected to $2 \mathrm{~Hz}$ sinusoidal loading from $0.1-1$

$50 \quad \mathrm{~N}(1 \%-20 \%$ of failure force, $\mathrm{n}=4)$ or $1-3 \mathrm{~N}(20-70 \%$ of failure force, $\mathrm{n}=5)$. To investigate

51 molecular level damage localization in the entheses, additional samples were loaded to 10,000

52 cycles $(n=3), 40,000$ cycles $(n=3)$, and to failure $(>50,000$ cycles, $n=5)$ using the second protocol

$53 \quad(20-70 \% \max$ failure force).

54 Enthesis structural properties, such as failure load (referred to as strength in text),

55 stiffness, and work to failure (area under the curve through failure load, referred to as toughness

56 in text) were determined from load-deformation curves. Stiffness was calculated by a MATLAB

57 (Matlab2019a, MathWorks) custom algorithm that identifies the best fitting line within a

58 sufficient bin width (i.e., remove data below $10 \%$ of max load and above $95 \%$ of max load) by

59 implementing the random sample correlation (RANSAC) technique (58).

50

51 Contrast enhanced and conventional micro computed tomography (microCT) imaging

52 Simultaneous visualization of soft and hard tissues of tendon enthesis samples were achieved by

53 staining samples with 5\% mercury chloride solution prior to scanning with microCT. A 5\%

54 mercury chloride solution was prepared fresh for each experiment day by dissolving Mercury (II)

55

56

57

58

59 chloride ( $\mathrm{HgCl}$, Sigma-Aldrich) in distilled and de-ionized water (MilliQ water,

MilliporeSigma) at room temperature until the saturation was achieved. Tendon enthesis samples, either intact or post-mechanical testing, were submerged in this solution for 24 hours and washed three times in distilled and de-ionized water for 10 minutes each before they were imaged with microCT (Skyscan 1272, Bruker). 
70 We used the same preparations and scan settings when visualizing enthesis samples with both

71 conventional and contrast enhanced microCT. To prepare for scanning, distal end of

72 supraspinatus-humerus unit were embedding in 2\% agarose (Sigma-Aldrich) and mounted in the

73 scanning chamber, so that tendon enthesis specimens were hung loosely and in line with the

74 scanning axis. To visualize enthesis samples at specific angles, we used 3D printed fixtures that

75 fixed the samples in the appropriate position when they were mounted in the scanning chamber.

76 Scans were performed with $60 \mathrm{kVp}, 166 \mathrm{uA}$, and $\mathrm{Al} 0.5 \mathrm{~mm}$ filters with isometric resolution of 2.5

$77 \mu \mathrm{m}$. To visualize enthesis insertions and failure surfaces, high resolution images were obtained at

$78 \quad 0.75 \mu \mathrm{m}$ resolution, while for whole joint imaging images were obtained at $5 \mu \mathrm{m}$ resolution. The

79 acquired microCT data were reconstructed with the software (nRecon, Bruker) provided with the

80 CT scanner using alignment optimization and beam-hardening correction. The reconstructed

81 image data was visualized with built-in program (DataViewer and CTvox, Bruker).

82

83

84

85

86

87

\section{Scanning Electron Microscopy (SEM)}

Failed tendon enthesis samples $(n=10)$ were dried at $37^{\circ} \mathrm{C}$, fixed on SEM aluminum pin mounts using carbon tape and silver paint and carbon-coated (30 nm). Prepared samples were imaged by scanning electron microscope (FEGSEM, Quanta 250F, FEI Company, Hillsboro, OR, USA) in backscattered electron mode using a concentric backscattered detector and acceleration voltages of 5-15 KV at a working at different magnifications from 250x to 20,000x. SEM was carried out using facilities at the University Service Centre for Transmission Electron Microscopy, TU Wien, Austria.

Tendon cross-sectional area, mineralized fibrocartilage area, footprint area, insertion area, and failure area determination 
94 Conventional and contrast enhanced microCT scans of murine tendon enthesis samples were 95 analyzed to determine minimal tendon cross-sectional area, mineralized fibrocartilage (MFC)

96 area, enthesis footprint area, insertion area, and failure area. The minimum tendon cross-sectional

97 area and mineralized fibrocartilage area for each sample was determined from conventional

98 microCT scans that were performed on samples prior to mechanical testing (or prior to staining

99 with $\mathrm{HgCl}_{2}$ ) and analyzed via built-in image processing algorithms (CTAn, Bruker). Minimum

jo cross-sectional tendon area was determined by thresholding the transverse slices through the

31 tendon, calculating the area encompassing the tendon, and selecting the smallest area of a tendon

J2 that is within $500 \mu \mathrm{m}$ from the tendon insertion site. MFC volume was determined by contouring,

3 thresholding, and integrating all the areas of MFC from sagittal slices of humeral head. Since the

J4 absorption coefficients of the MFC was in between that of tendon and bone, and did not change

55 significantly between samples, a single range of threshold values was selected to identify and

06 estimate volume of the MFC.

37 Apparent footprint area, insertion area, and failure area were estimated using $\mathrm{HgCl}_{2}$ stained

08 contrast enhanced microCT images of enthesis samples, as the imaging technique allows for

99 differential absorbance coefficients between each tissue selected. Since the regions of interest

10 were along the curved volume (i.e., humeral head), we developed a custom semi-automated

11 MATLAB (Matlab2019a, MathWorks) routine that calculates the overlapping polyhedron surface

12 meshes from two arbitrary volumes (e.g., humeral head and tendon enthesis) from the same

13 imaging dataset. The first region represents the surface of the bone: either the surface of the

14 humeral head (for calculating footprint area or insertion area), or the surface of avulsed pieces

15 (for calculating failure area). This region was obtained by thresholding and semi-automatically

16 contouring via shrink-wrapping algorithm built-in to the manufacturers' imaging processing

17 software (CTan, Bruker). The second region for calculating footprint area or insertion area

18 represents volume of the tendon enthesis that intersects with the surface of the humeral head 
19 along the edge of the tendon attachment. The second region for calculating the failure area

20 represents a volume that contains only the fractured surface of the avulsed piece. The edges of the

21 second region for in both cases were determined visually by an experienced researcher by

22 manually contouring appropriately slices for each region of interest. The output volume sets were

23 triangularly meshed to determine the surface area between the overlapping volumes.

\section{Collagen damage visualization}

26 Unloaded and loaded tendon enthesis samples allocated for analyzing molecular-level collagen

27 damage were stained with F-CHP (3 Helix) and visualized via fluorescence microscopy. Post

28 mechanical testing, samples were first secured and fixed at their appropriate displacements with

$294 \%$ paraformaldehyde (PFA, Fisher Sci) overnight. Tendon enthesis samples were washed 3 times

30 in PBS for 10 min each at room temperature. After washing, each tendon enthesis sample was

31 placed in a tube containing $450 \mu \mathrm{l}$ of PBS solution. F-CHP staining protocol was adapted from

32 what have described previously in staining rat tendon fascicles (31). CF-CHP stock solution (150

$33 \mu \mathrm{M}$ ) was heated at $80{ }^{\circ} \mathrm{C}$ for 10 min to thermally dissociate trimeric CHP to a monomeric state

34 and quenched in ice bath for approximately 20 seconds to prevent artificial thermal damage to

35 samples. $50 \mu \mathrm{l}$ of monomeric CF-CHP were then added to a tube containing tendon enthesis

36 sample, resulting in a final F-CHP concentration of $15 \mu \mathrm{M}$. Samples were incubated for overnight

37 at $4^{\circ} \mathrm{C}$ and washed in PBS 3 times for $30 \mathrm{~min}$ in a room temperature to remove any unbound F-

38 CHP molecules. Stained samples were mounted on a glass slide and imaged and captured using

39 an automated ZEISS Microscope (10x objective, excitation at 488nm channel). Images were

40 captured by CCD camera using the built-in image acquisition and stitching features and analyzed

41 with ZEN lite software (ZEISS). 
Positional recruitment model

We consider $N$ linear elastic fibers of thickness $t$, each spaced a distance $s$ apart, beginning with a fiber that is immediately to the left of a circular bone ridge of radius $R$. When the grip is turned at an angle $\theta$ to represent positional change, fibers are stretched in that direction. We incorporated three assumptions in building the positional recruitment model as were suggested by the contrastenhanced imaging results: (1) the outer (bursal side) fibers longer than the inner (articular side) fibers, making the innermost fiber $(n=1)$ shortest; (2) tendon fibers are buckled at high angles of abduction; (3) to simplify, fibers were assumed to be elastic, brittle, and frictionless. During loading, fibers engage, re-orient, and, depending on loading direction, contact its neighbor fibers (or the humeral head) due to curvature of the humeral head (Fig.3a, FigS5). The contact point is determined for each fiber at $\vec{r}_{1}^{n}=R^{n}\left(-\cos \phi_{1}^{n} \hat{\imath}+\sin \phi_{1}^{n} \hat{\jmath}\right)$, where the radius of the centerline of the wrapped fiber is $R^{n}=R+(n-0.5) t$ and the contact angle is $\cos \phi_{1}^{n}=R^{n} / x_{0}^{n}$. The angle $\phi_{2}^{n}$ at which contact is lost is determined by the innermost fiber, which always stays in tension. Contact is lost at the point $\vec{r}_{2}^{n}(t)$ at which the unit vector between $\vec{r}_{2}^{n}(t)$ and the connection point on the grip for the strand, $\vec{r}_{3}^{n}(t)$, is tangent to the circle formed by the midline of fiber $n$. Using this we can determine the maximum length of a fiber when it is engaged:

$$
L_{e n}^{n}(t)=\left\|\vec{r}_{1}^{n}-\vec{r}_{0}^{n}\right\|+\left(\phi_{2}^{n}(t)-\phi_{1}^{n}\right) R^{n}+\left\|\vec{r}_{3}^{n}(t)-\vec{r}_{2}^{n}(t)\right\|
$$

when a fiber is engaged and contact the bone ridge. If a fiber is engaged, but does not contact the bone ridge (when $\left.\phi_{2}^{n}(t)<\phi_{1}^{n}\right)$ :

$$
L_{e n}^{n}(t)=\left\|\vec{r}_{3}^{n}(t)-\vec{r}_{0}^{n}\right\|
$$

We generated load-displacement curves from this position dependent fiber kinematic model.

Expanded details on the positional recruitment model can be found in the supplemental document (Supplementary Text). 


\section{Removal of extracellular matrix components}

58 Glycosaminoglycans (GAGs) from the tendon enthesis samples were chemically digested by 59 adapting a chondroitinase $\mathrm{ABC}(\mathrm{ChABC})$ treatment protocol, which is known to degrade $\mathrm{GAG}$

70 chains from tendon (59). After conducting a series of concentration and time dependent tests

71 (results not shown), we determined that $0.5 \mathrm{U} / \mathrm{mL}$ was an optimum concentration for ChABC for

72 digesting GAGs from tendon enthesis samples. In this protocol, whole samples (humerus-

73 supraspinatus tendon-supraspinatus muscle units) were incubated for 5 days in $2 \mathrm{~mL}$ of $0.5 \mathrm{U} / \mathrm{mL}$

74 chABC buffered solution (the buffer solution consists of $50 \mathrm{mM}$ Tris, $60 \mathrm{mM}$ sodium acetate,

$75 \quad 0.02 \%$ bovine serum albumin). After 5 days, digested samples were washed in $1 \times$ PBS solution 3

76 times for 30 minutes before subjecting them to microCT imaging and quasi-static mechanical

77 testing. To evaluate the efficiency of ChABC treatment, we performed histological analysis on

78 some samples instead of mechanical testing. These samples $(n=2)$ were fixed in $4 \%$

79 paraformaldehyde for 24 hours, decalcified in formic acid (StatLab, Immunocal), dehydrated in

$80 \quad 70 \%$ ethanol, and embedded in paraffin. $5 \mu \mathrm{m}$ thickness paraffin sections were stained with

81 Alcian blue using manufacturers protocol (Alcian Blue Stain Kit, Abcam) and imaged via bright

82 field microscopy with $10 \times$ and $40 \times$ objectives.

83 Mineral was chemically removed from the tendon enthesis samples by incubating in $5 \mathrm{~mL}$ formic

84 acid (Immunocal, StatLab) for 72 hours. Samples were washed in 1xPBS solution 3 times for 30

85 minutes before subjecting them to microCT imaging to confirm that all the mineral components

86 were chemically digested, and then quasi-static mechanical testing.

\section{In vivo degeneration models}

89 10-week old C57BL6/J mice ( $\mathrm{n}=10$ /group, Jackson Laboratories) were subjected to two in vivo

$90 \quad$ loading models, where the supraspinatus muscle activity was modulated to modify supraspinatus

91 tendon enthesis loading environment. (1) Underuse-degeneration (underuse) was induced via 
muscle paralysis by bilaterally injecting $0.2 \mathrm{U}(0.1 \mathrm{U} / 10 \mu \mathrm{l}$ per $100 \mathrm{~g}$ of body weight) of botulinum toxin into the supraspinatus muscles. After injections, mice were allowed to free cage activity for 4 weeks. (2) Overuse-degeneration was achieved using downhill treadmill running (overuse) with an initial rate of $17 \mathrm{~cm} / \mathrm{s}$ for 10 minutes followed by $25 \mathrm{~cm} / \mathrm{s}$ for $40 \mathrm{~min}$ each day at a decline of 15 degrees, 5 days a week, for 4 weeks (60). To acclimate the mice to treadmill exercises, 1 week prior to the overuse protocol, mice underwent training: exercising for each day for 10 minutes at $17 \mathrm{~cm} / \mathrm{s}$ for 5 days followed by 2 days of rest. For both in vivo models, after 4 weeks since the protocol initiation, mice were euthanized and their supraspinatus tendon enthesis were harvested, soaked in PBS, and stored at $-20^{\circ} \mathrm{C}$.

\section{Bone morphometry and individual trabecula segmentation (ITS) analysis}

Bone morphometry parameters, such as bone volume/total volume (BV/TV), trabecular thickness (Tb.Th.), and trabecular spacing (Tb.Sp.) of the trabecular bone, as well as parameters obtained from ITS analysis were determined using pre-mechanical testing scans of tendon enthesis $(5.0 \mu \mathrm{m}$ resolution). Reconstructed images were first contoured by an experienced user (MG and AA) to only include humeral head proximal to the growth plate as the region of interest (ROI). The ROI were then evaluated using a segmentation algorithm that separates cortical and trabecular bone (CTAn, Bruker). Segmented trabecular images were subjected to subsequent microstructural ITS analysis, where trabecular microstructures were decomposed to individual rod-and-plate-based trabecular microstructural parameters (40). In short, the thresholded trabecular bone images were reduced to topology-preserved structural skeletons using digital topological analysis-based skeletonization technique. Each skeletal voxel was then recovered to original topology using an iterative reconstruction method, while classifying whether the resulting trabecular structure belong to either a trabecular plate (surface) or a trabecular rod (curve) using digital topological classification methodology. Microstructural trabecular network and morphology parameters, such as plate-to-rod ratio (PR ratio), rod and plate bone volume fraction (rBV/TV and $\mathrm{pBV} / \mathrm{TV}$ ), 
18 number density (rTb.N and pTb.N), and thickness (rTb.Th and pTb.Th) were then evaluated from

19 resultant three-dimensional rod-and-plate classified trabecular morphology. The angular

20 orientational analysis was also performed by evaluating each rod-and-plate angle with respect to

21 perpendicular to the loading axis corresponding to 90 degrees abduction. The average angular

22 distribution for each sample was normalized by the total trabecular volume within each sample's

23 humeral head.

\section{Statistical Analysis}

26 Tendon enthesis characteristics, biomechanics results, failure properties, and bone morphometry

27 results were compared between treatment groups using ANOVA and specific differences from

28 control conditions were determined using Dunnett's multiple comparisons test. $\mathrm{P}<0.05$ was

29 considered significant. Failure properties were correlated to bone morphometry outcomes using

30 Pearson correlation. All statistical analyses were performed using Prism 9 (GraphPad). All data

31 shown as mean \pm standard deviation and results from Pearson correlation were expressed using

32 the color map. 


\section{References}

34 1. Z. Yin, F. Hannard, F. Barthelat, Impact-resistant nacre-like transparent materials. Science.

364, 1260-1263 (2019).

2. Y. Liu, N. A. Fleck, V. S. Deshpande, A. Srivastava, High fracture toughness microarchitectured materials. J. Mech. Phys. Solids. 143, 104060 (2020).

3. N. A. Fleck, V. S. Deshpande, M. F. Ashby, Micro-architectured materials: Past, present and future. Proc. R. Soc. A Math. Phys. Eng. Sci. 466, 2495-2516 (2010).

4. M. Mirkhalaf, A. K. Dastjerdi, F. Barthelat, Overcoming the brittleness of glass through bio-inspiration and micro-architecture. Nat. Commun. 5, 3166 (2014).

5. F. Barthelat, Z. Yin, M. J. Buehler, Structure and mechanics of interfaces in biological materials. Nat. Rev. Mater. 1, 16007 (2016).

6. F. Barthelat, Architectured materials in engineering and biology: Fabrication, structure, mechanics and performance. Int. Mater. Rev. 60, 412-430 (2015).

7. R. O. Ritchie, The conflicts between strength and toughness. Nat. Mater. 10, 817-822 (2011).

8. G. M. Genin, S. Thomopoulos, The tendon-to-bone attachment: Unification through disarray. Nat. Mater. 16, 607-608 (2017).

9. L. Rossetti, L. A. Kuntz, E. Kunold, J. Schock, K. W. Müller, H. Grabmayr, J. StolbergStolberg, F. Pfeiffer, S. A. Sieber, R. Burgkart, A. R. Bausch, The microstructure and micromechanics of the tendon-bone insertion. Nat. Mater. 16, 607-608 (2017).

10. S. Thomopoulos, G. R. Williams, J. A. Gimbel, M. Favata, L. J. Soslowsky, Variation of biomechanical, structural, and compositional properties along the tendon to bone insertion site. J. Orthop. Res. 21, 413-419 (2003).

11. A. C. Deymier-Black, J. D. Pasteris, G. M. Genin, S. Thomopoulos, Allometry of the Tendon Enthesis: Mechanisms of Load Transfer Between Tendon and Bone. J. Biomech. 
Eng. 137, 111005 (2015).

12. S. P. Lake, K. S. Miller, D. M. Elliott, L. J. Soslowsky, Effect of fiber distribution and realignment on the nonlinear and inhomogeneous mechanical properties of human supraspinatus tendon under longitudinal tensile loading. J. Orthop. Res. 27, 1596-602 (2009).

13. S. Thomopoulos, J. P. Marquez, B. Weinberger, V. Birman, G. M. Genin, Collagen fiber orientation at the tendon to bone insertion and its influence on stress concentrations. $J$. Biomech. 39, 1842-1851 (2006).

14. G. M. Genin, A. Kent, V. Birman, B. Wopenka, J. D. Pasteris, P. J. Marquez, S. Thomopoulos, Functional grading of mineral and collagen in the attachment of tendon to bone. Biophys. J. 97, 976-985 (2009).

15. Y. Hu, V. Birman, A. Demyier-Black, A. G. Schwartz, S. Thomopoulos, G. M. Genin, Stochastic interdigitation as a toughening mechanism at the interface between tendon and bone. Biophys. J. 108, 431-437 (2015).

16. A. C. Deymier, A. G. Schwartz, Z. Cai, T. L. Daulton, J. D. Pasteris, G. M. Genin, S. Thomopoulos, The multiscale structural and mechanical effects of mouse supraspinatus muscle unloading on the mature enthesis. Acta Biomater. 83, 302-313 (2019).

17. M. J. Buehler, Molecular nanomechanics of nascent bone: Fibrillar toughening by mineralization. Nanotechnology. 18, 295102 (2007).

18. M. S. Rashid, C. Cooper, J. Cook, D. Cooper, S. G. Dakin, S. Snelling, A. J. Carr, Increasing age and tear size reduce rotator cuff repair healing rate at 1 year. Acta Orthop. 88, 606-611 (2017).

19. L. M. Galatz, C. M. Ball, S. A. Teefey, W. D. Middleton, K. Yamaguchi, The Outcome and Repair Integrity of Completely Arthroscopically Repaired Large and Massive Rotator Cuff Tears. J. Bone Jt. Surg. - Ser. A. 86, 219-224 (2004). 
20. J. Sartori, H. Stark, Tracking tendon fibers to their insertion - a 3D analysis of the Achilles tendon enthesis in mice. Acta Biomater. 120, 146-155 (2020).

21. Y. Liu, V. Birman, C. Chen, S. Thomopoulos, G. M. Genin, Mechanisms of Bimaterial Attachment at the Interface of Tendon to Bone. J. Eng. Mater. Technol. 133, 011006 (2010).

22. F. Saadat, A. C. Deymier, V. Birman, S. Thomopoulos, G. M. Genin, The concentration of stress at the rotator cuff tendon-to-bone attachment site is conserved across species. $J$. Mech. Behav. Biomed. Mater. 62, 24-32 (2016).

23. H. M. Shaw, M. Benjamin, Structure-function relationships of entheses in relation to mechanical load and exercise: Review. Scand. J. Med. Sci. Sport. 17, 303-315 (2007).

24. J. M. Weiss, A. Arkader, L. M. Wells, T. J. Ganley, Rotator cuff injuries in adolescent athletes. J. Pediatr. Orthop. Part B. 22, 133-137 (2013).

25. J. Y. Jeong, S. K. Min, K. M. Park, Y. B. Park, K. J. Han, J. C. Yoo, Location of Rotator Cuff Tear Initiation: A Magnetic Resonance Imaging Study of 191 Shoulders. Am. J. Sports Med. 46, 649-655 (2018).

26. H. M. Kim, N. Dahiya, S. A. Teefey, W. D. Middleton, G. Stobbs, K. Steger-May, K. Yamaguchi, J. D. Keener, Location and initiation of degenerative rotator cuff tears: An analysis of three hundred and sixty shoulders. J. Bone Jt. Surg. - Ser. A. 92, 829-839 (2010).

27. K. Yamaguchi, K. Ditsios, W. D. Middleton, C. F. Hildebolt, L. M. Galatz, S. A. Teefey, The demographic and morphological features of rotator cuff disease: A comparison of asymptomatic and symptomatic shoulders. J. Bone Jt. Surg. - Ser. A. 88, 1699-704 (2006).

28. M. I. Danto, S. L. Woo, The mechanical properties of skeletally mature rabbit anterior cruciate ligament and patellar tendon over a range of strain rates. J. Orthop. Res. 11, 58-67 (1993). 
29. H. A. Lynch, W. Johannessen, J. P. Wu, A. Jawa, D. M. Elliott, Effect of Fiber Orientation and Strain Rate on the Nonlinear Uniaxial Tensile Material Properties of Tendon. $J$. Biomech. Eng. 125, 726 (2003).

30. J. L. Zitnay, G. S. Jung, A. H. Lin, Z. Qin, Y. Li, S. M. Yu, M. J. Buehler, J. A. Weiss, Accumulation of collagen molecular unfolding is the mechanism of cyclic fatigue damage and failure in collagenous tissues. Sci. $A d v .6$, eaba2795 (2020).

31. J. L. Zitnay, Y. Li, Z. Qin, B. H. San, B. Depalle, S. P. Reese, M. J. Buehler, S. M. Yu, J. A. Weiss, Molecular level detection and localization of mechanical damage in collagen enabled by collagen hybridizing peptides. Nat. Commun. 8, 14913 (2017).

32. S. P. McCully, N. Kumar, M. D. Lazarus, A. R. Karduna, Internal and external rotation of the shoulder: Effects of plane, end-range determination, and scapular motion. J. Shoulder Elb. Surg. 14, 605-610 (2005).

33. M. D. Newton, A. A. Davidson, R. Pomajzl, J. Seta, M. D. Kurdziel, T. Maerz, The influence of testing angle on the biomechanical properties of the rat supraspinatus tendon. J. Biomech. 49, 4159-4163 (2016).

34. K. Lindblom, On pathogenesis of ruptures of the tendon aponeurosis of the shoulder joint. Acta radiol. 20, 564-577 (1939).

35. C. Y. Huang, V. M. Wang, R. J. Pawluk, J. S. Bucchieri, W. N. Levine, L. U. Bigliani, V. C. Mow, E. L. Flatow, Inhomogeneous mechanical behavior of the human supraspinatus tendon under uniaxial loading. J. Orthop. Res. 23, 924-930 (2005).

36. H. Ouellette, J. Labis, M. Bredella, W. E. Palmer, K. Sheah, M. Torriani, Spectrum of shoulder injuries in the baseball pitcher. Skeletal Radiol. 37, 491-498 (2008).

37. O. A. Tertuliano, J. R. Greer, The nanocomposite nature of bone drives its strength and damage resistance. Nat. Mater. 15, 1195-1202 (2016).

38. C. Gerber, J. G. Snedeker, D. Baumgartner, A. F. Viehöfer, Supraspinatus tendon load 
during abduction is dependent on the size of the critical shoulder angle: A biomechanical analysis. J. Orthop. Res. 32, 952-957 (2014).

39. Y. Liu, S. Thomopoulos, C. Chen, V. Birman, M. J. Buehler, G. M. Genin, Modelling the mechanics of partially mineralized collagen fibrils, fibres and tissue. J. R. Soc. Interface. 11, 20130835 (2014).

40. A. C. Deymier, Y. An, J. J. Boyle, A. G. Schwartz, V. Birman, G. M. Genin, S. Thomopoulos, A. H. Barber, Micro-mechanical properties of the tendon-to-bone attachment. Acta Biomater. 56, 25-35 (2017).

41. I. M. Basalo, F. H. Chen, C. T. Hung, G. A. Ateshian, Frictional response of bovine articular cartilage under creep loading following proteoglycan digestion with chondroitinase ABC. J. Biomech. Eng. 128, 131-4 (2006).

42. O. G. Andriotis, S. Desissaire, P. J. Thurner, Collagen Fibrils: Nature's Highly Tunable Nonlinear Springs. ACS Nano. 12, 3671-3680 (2018).

43. D. R. Carter, Calcif. Tissue Int., in press, doi:10.1007/BF02406129.

44. L. B. Meakin, J. S. Price, L. E. Lanyon, The contribution of experimental in vivo models to understanding the mechanisms of adaptation to mechanical loading in bone. Front. Endocrinol. (Lausanne). 5, 154 (2014).

45. A. M. Tatara, J. H. Lipner, R. Das, H. M. Kim, N. Patel, E. Ntouvali, M. J. Silva, S. Thomopoulos, The role of muscle loading on bone (re)modeling at the developing enthesis. PLoS One. 9, e97375 (2014).

46. X. S. Liu, P. Sajda, P. K. Saha, F. W. Wehrli, G. Bevill, T. M. Keaveny, X. E. Guo, Complete volumetric decomposition of individual trabecular plates and rods and its morphological correlations with anisotropic elastic moduli in human trabecular bone. $J$. Bone Miner. Res. 23, 223-35 (2008).

47. E. R. Cadet, J. W. Hsu, W. N. Levine, L. U. Bigliani, C. S. Ahmad, The relationship 
between greater tuberosity osteopenia and the chronicity of rotator cuff tears. J. Shoulder Elb. Surg. 17, 73-77 (2008).

48. F. Barthelat, H. D. Espinosa, An experimental investigation of deformation and fracture of nacre-mother of pearl. Exp. Mech. 47, 311-324 (2007).

49. V. Imbeni, J. J. Kruzic, G. W. Marshall, S. J. Marshall, R. O. Ritchie, The dentin-enamel junction and the fracture of human teeth. Nat. Mater. 4, 229-232 (2005).

50. F. Marin, N. Le Roy, B. Marie, The formation and mineralization of mollusk shell. Front. Biosci. - Sch. 4, 1099-125 (2012).

51. K. Livanov, H. Jelitto, B. Bar-On, K. Schulte, G. A. Schneider, D. H. Wagner, Tough alumina/polymer layered composites with high ceramic content. J. Am. Ceram. Soc. 98, 1285-1291 (2015).

52. B. Han, K. K. Qin, B. Yu, Q. C. Zhang, C. Q. Chen, T. J. Lu, Design optimization of foamreinforced corrugated sandwich beams. Compos. Struct. 130, 51-62 (2015).

53. S. Khandelwal, T. Siegmund, R. J. Cipra, J. S. Bolton, Transverse loading of cellular topologically interlocked materials. Int. J. Solids Struct. 49, 2394-2403 (2012).

54. M. Carlesso, R. Giacomelli, T. Krause, A. Molotnikov, D. Koch, S. Kroll, K. Tushtev, Y. Estrin, K. Rezwan, Improvement of sound absorption and flexural compliance of porous alumina-mullite ceramics by engineering the microstructure and segmentation into topologically interlocked blocks. J. Eur. Ceram. Soc. 33, 2549-2558 (2013).

55. M. B. Schmidt, V. C. Mow, L. E. Chun, D. R. Eyre, Effects of proteoglycan extraction on the tensile behavior of articular cartilage. J. Orthop. Res. 8, 353-63 (1990).

56. S. Cavelier, A. K. Dastjerdi, M. D. McKee, F. Barthelat, Bone toughness at the molecular scale: A model for fracture toughness using crosslinked osteopontin on synthetic and biogenic mineral substrates. Bone 110, 304-311 (2018).

57. I. Kurtaliaj, M. Golman, A. C. Abraham, S. Thomopoulos, Biomechanical Testing of 
Murine Tendons. J. Vis. Exp. (2019), doi:10.3791/60280.

58. S. W. Linderman, M. Golman, T. R. Gardner, V. Birman, W. N. Levine, G. M. Genin, S. Thomopoulos, Enhanced tendon-to-bone repair through adhesive films. Acta Biomater. 70, 165-176 (2018).

59. F. Fang, S. P. Lake, Multiscale mechanical integrity of human supraspinatus tendon in shear after elastin depletion. J. Mech. Behav. Biomed. Mater. 63 443-455 (2016).

60. A. C. Abraham, S. A. Shah, M. Golman, L. Song, X. Li, I. Kurtaliaj, M. Akbar, N. L. Millar, Y. Abu-Amer, L. M. Galatz, S. Thomopoulos, Targeting the NF-kB signaling pathway in chronic tendon disease. Sci. Transl. Med. 11, eaav4319 (2019).

61. M. M. Panjabi, A. A. White, W. O. Southwick, Mechanical properties of bone as a function of rate of deformation. J. Bone Joint Surg. Am. 55, 322-30 (1973).

62. T. M. Wright, W. C. Hayes, Tensile testing of bone over a wide range of strain rates: effects of strain rate, microstructure and density. Med. Biol. Eng. 14, 671 (1976).

\section{Acknowledgments}

The project was funded by National Institute of Health (NIH) U01-EB016422 and R01AR055580. We acknowledge Fei Fang with the help on in vivo degeneration models. We acknowledge Vedran Nedelovski, Amir Davood Elmi and Martin Handelshauser for providing Electron Microscopy images of failed tendon enthesis, which were obtained at the University Service Centre for Transmission Electron Microscopy, TU Wien, Austria.

Author contributions: M.G., G.M.G., S.T., and V.B., designed the research. M.G., A.C.A., I.K., and B.P.M., developed protocols and performed microcomputed tomography. A.G.S. obtained histological slides. M.G. carried out mechanical testing, confocal microscopy, and contrast- 
08 enhanced microcomputed tomography. P.J.T. obtained scanning electron microscopy images.

39 Y.J.H., and X.E.G., performed ITS analysis. M.G., G.M.G., and S.T., analyzed the data and wrote

10 the paper. All authors reviewed and revised the manuscript.

11

12 Competing interests: Authors declare that they have no competing interests.

14 Data and materials availability: All data is available in the main text or the supplementary

15 materials. The custom codes used in this study, including the positional recruitment numerical

16 model, are available from the authors upon request. 

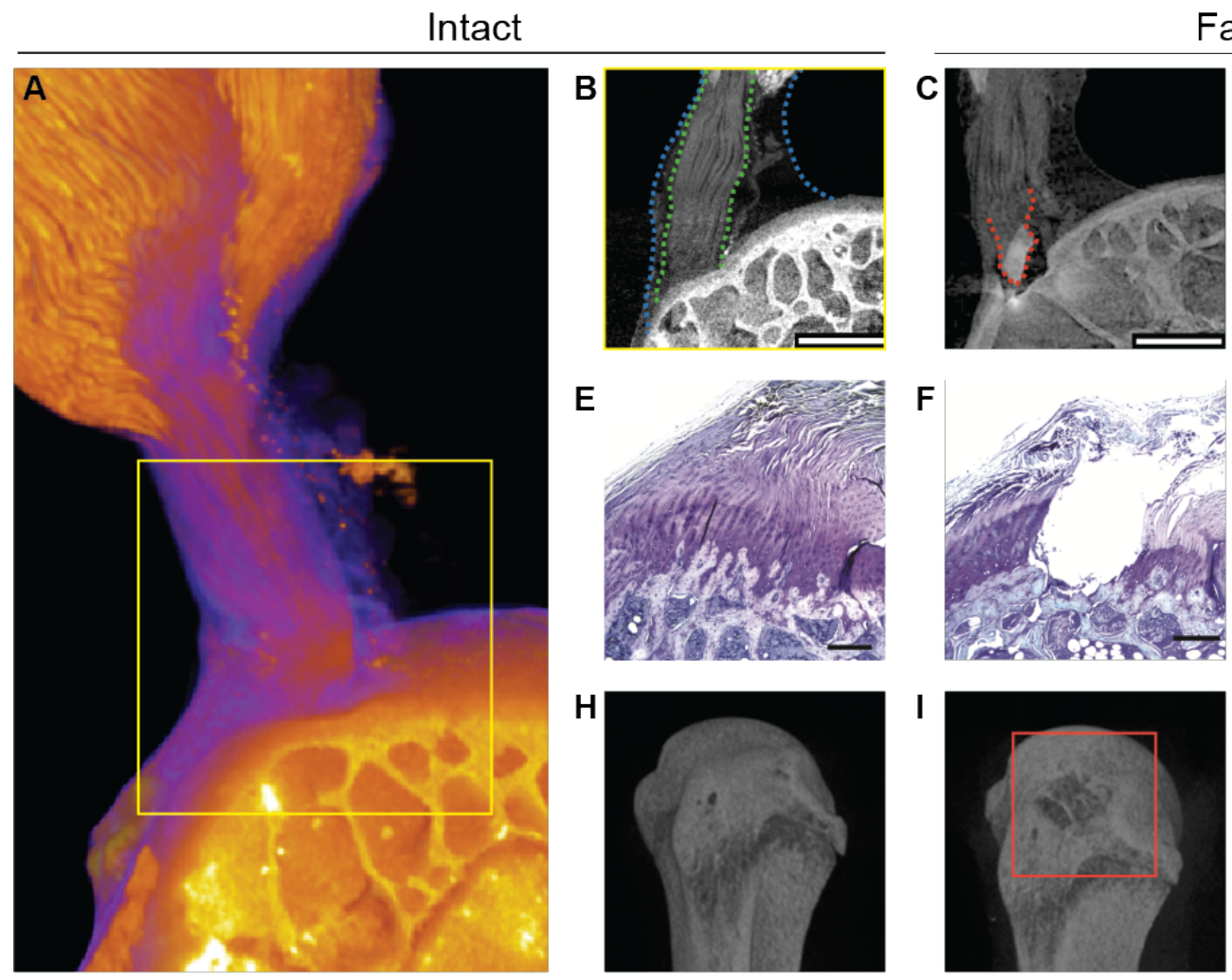

Failed
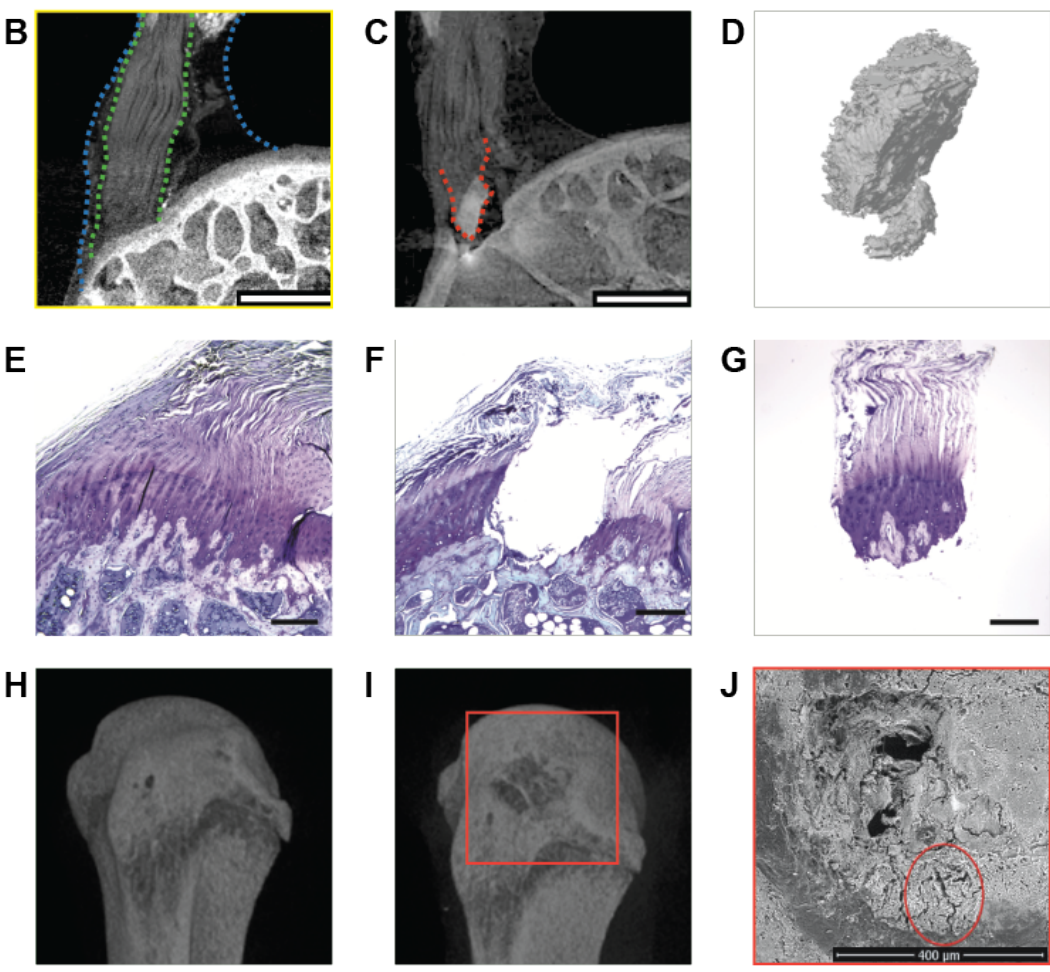

\section{G}
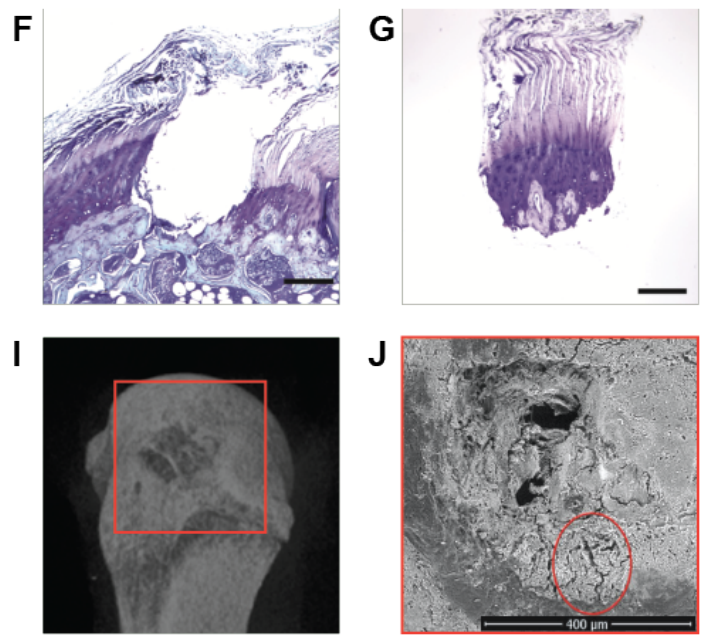

Fig. 1. The tendon enthesis exhibits a fibrous architectured material system that fails via

\section{bony avulsion under quasi-static loading.}

(A)-(C), Mercury (II) chloride-stained contrast enhanced high-resolution microCT imaging

$$
\text { revealed that, hidden within the well-known larger apparent attachment footprint area, is a }
$$

smaller, much denser primary insertion site where tendon fibers insert directly into the bone.

Imaging revealed that, under quasi-static loading, only $47.4+/-5.1 \%$ of the apparent attachment site was avulsed, revealing a previously unknown primary attachment. (A) Three-dimensional volume rendering of representative intact enthesis. (B) Magnified cross sectional view of yellow box in a; within blue dotted lines outline apparent enthesis and within green dotted lines outline dense primary insertion. (scale: $500 \mu \mathrm{m}$ ). (C) Post-failure imaging showing avulsed bony fragment at primary insertion site, outlined with a red dotted line. (scale: $500 \mu \mathrm{m}$ ). (D) Threedimensional representation of avulsed fragment showing portions of trabeculae at the failure site. 
31 (E)-(G), Histological sections of $(E)$ intact, and $(F)-(G)$ failed enthesis stained with toluidine blue 32 (scale: $250 \mu \mathrm{m})$. (H)-(I), Three-dimensional reconstruction from conventional microCT imaging 33 of a representative (H) intact and (I) failed enthesis sample. (J) Scanning electron microscopy of 34 the failure site showing crack propagation around the avulsion site, outlined by a red circle (scale $35400 \mu \mathrm{m})$. 
A

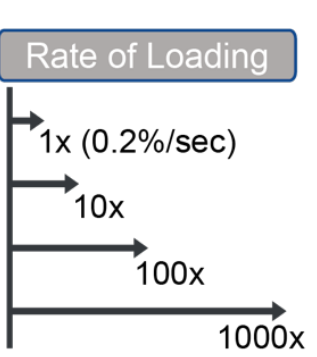

E

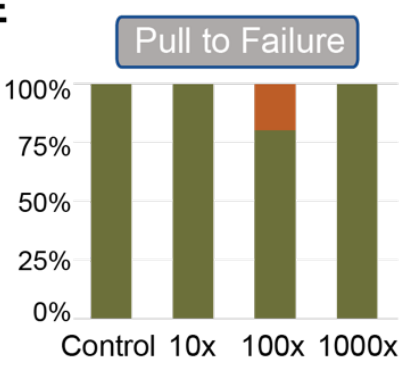

B

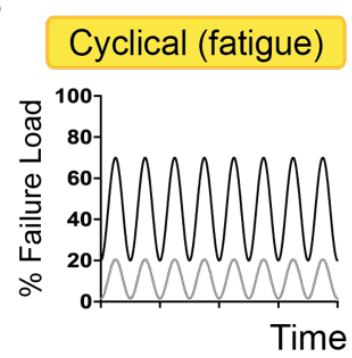

C

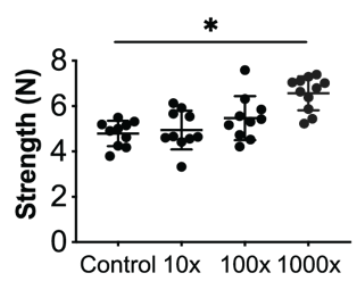

D

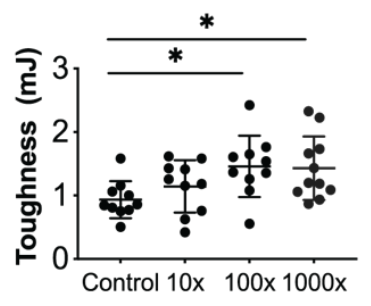

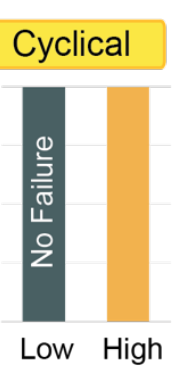
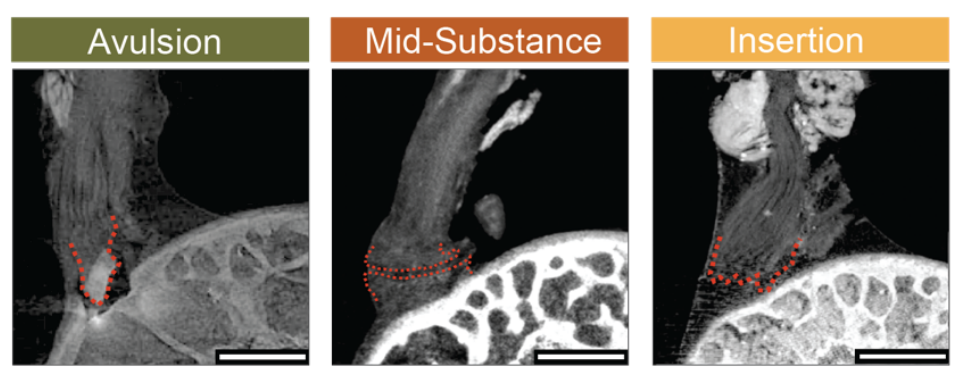

$\mathbf{F}$
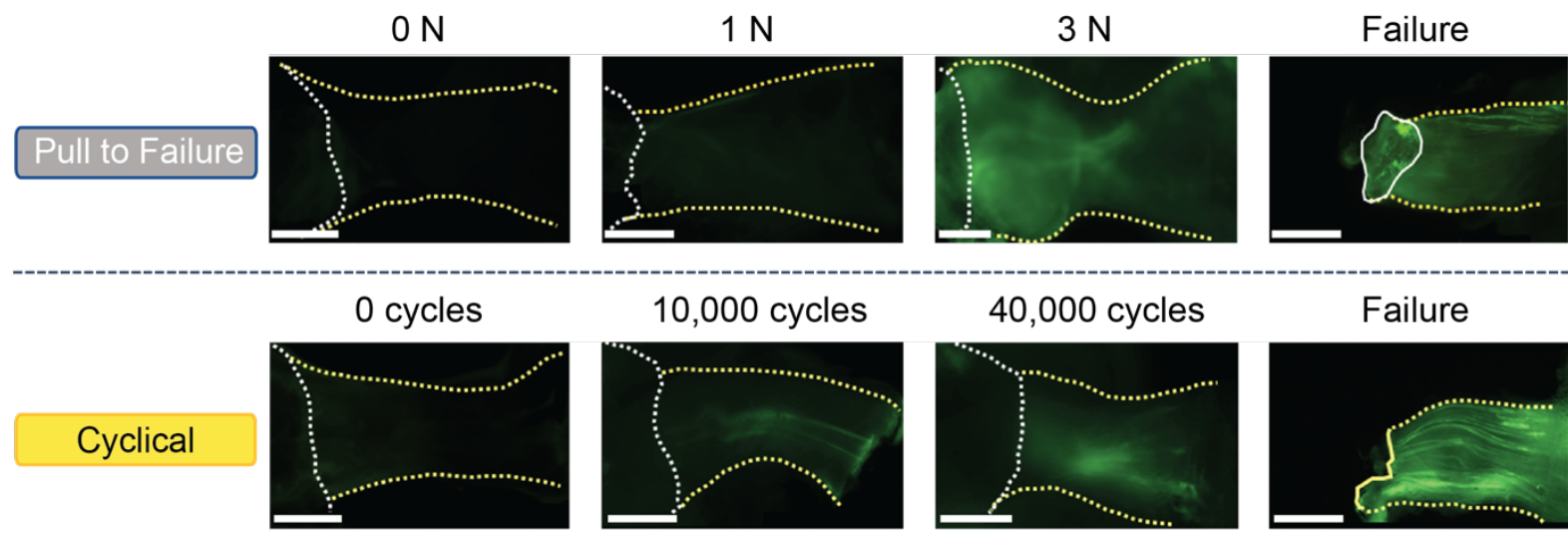

Failure

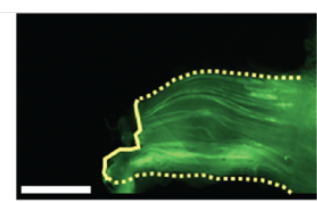

Fig. 2. Multiscale toughening mechanisms enable the entheses to exhibit distinct failure

\section{modes under varying loading conditions.}

(A)-(B), To examine effect of loading on failure mode, samples were loaded (A) across a range of loading rates to simulate acute injuries or (B) loaded cyclically to simulate degenerative loading. (C) Enthesis strength (i.e., failure load) and (D) enthesis toughness (i.e., energy absorption) increased with the loading rate. $(* * * * \mathrm{p}<0.0001, * \mathrm{p}<0.05$, ANOVA followed by the Dunnet's multiple comparison test). (E) There were three distinct failure modes, depending on the loading regime: bone avulsion, tendon mid-substance, and tendon-bone interface (insertion failure) (scale: $500 \mu \mathrm{m})$. Under monotonic loading, most samples failed by bony avulsion failures. Under "high"

cyclical loading (20\%-70\% failure force), all samples failed at the insertion. Under "low" cyclical loading (1\%-20\% failure force) samples did not fail, even after 100,000 cycles. (F) F-CHP 
49 fluorescence intensity, indicative of collagen damage accumulation, increased with the level of 50 applied load and with the number of cycles. For quasi-statically loaded samples (F, top), there 51 was little to no fluorescent signal in the low force group $(1 \mathrm{~N}-2 \mathrm{~N})$, followed by increased staining 52 near the attachment site at higher loads (3N and failure). For cyclically loaded samples ( $\mathrm{F}$, 53 bottom), F-CHP staining was initially concentrated in a few fibers near the tendon mid-substance 54 (10K-40K cycles) and ultimately propagated down the entire tendon in concentrated bands (scale: $55500 \mu \mathrm{m})$ 
A

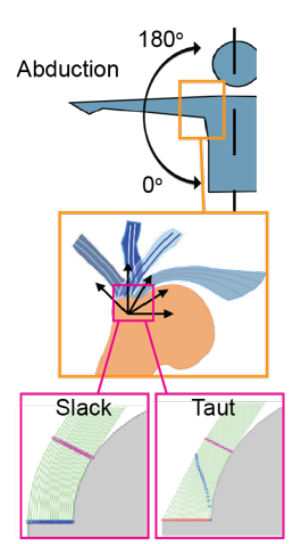

C
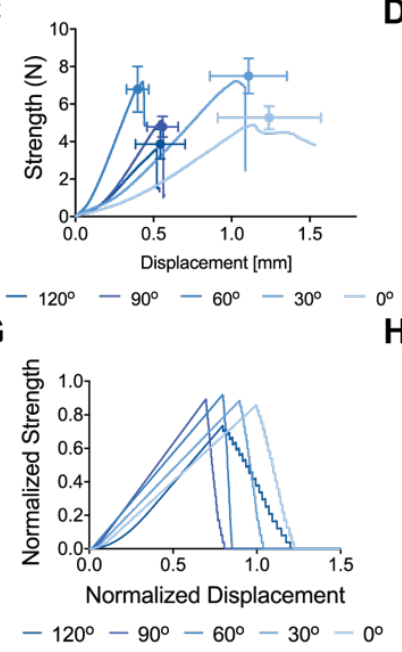

B
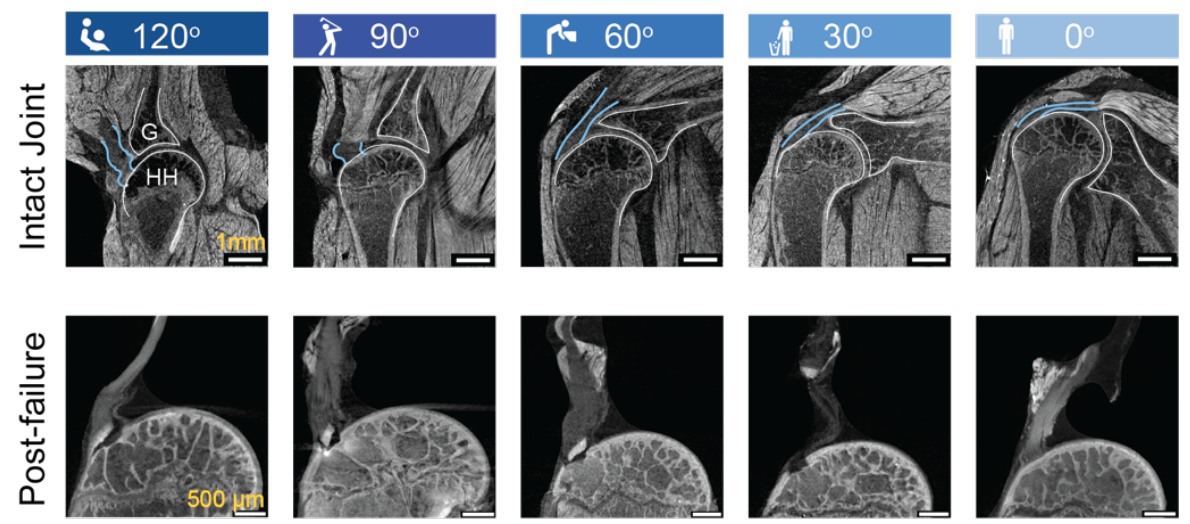

E
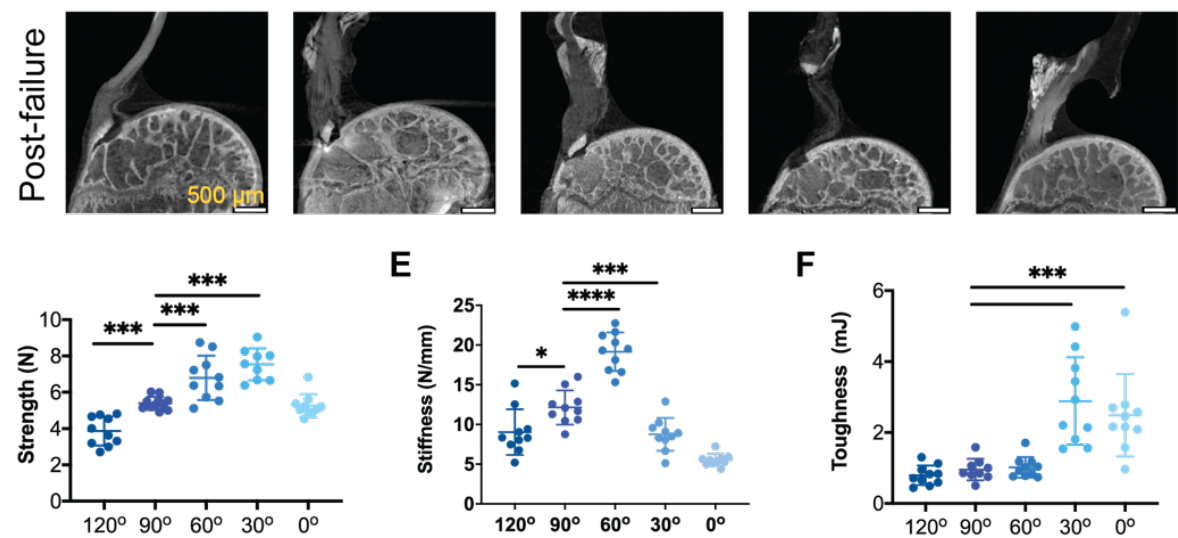

$\mathbf{F}$

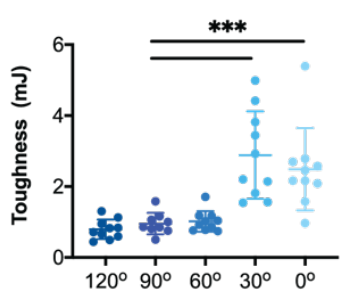

I

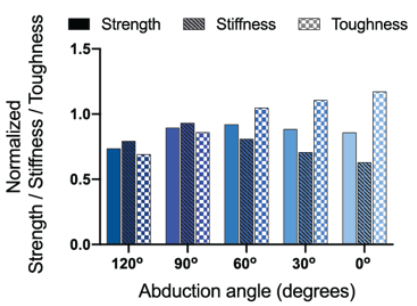

J

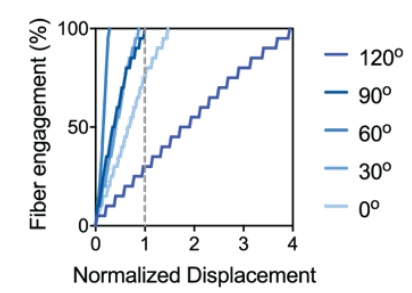

\section{Fig. 3. Multiscale toughening mechanisms enable the entheses to exhibit distinct failure}

\section{modes under varying loading conditions.}

(A) Samples were tested at varying angles of abduction (A, top) and a fiber recruitment model

was developed to examine structural and positional contributions to enthesis toughness (A,

bottom). (B) Contrast-enhanced microCT of intact (b, top row) and failed (b, bottom row) mouse

glenohumeral joints at each abduction angle (G: glenoid, HH: humeral head). The supraspinatus

tendon ( $\mathrm{b}$, top row, outlined in blue) was straight at low abduction angles $\left(0^{\circ}-30^{\circ}\right)$ and buckled at

high abduction angles $\left(90^{\circ}-120^{\circ}\right)$. (C)-(F), There were significant differences in the attachment mechanical behavior and failure properties when samples were tested quasistatically at varying

angles ex vivo (C, strength (failure force) vs. displacement plot; D, strength; E, stiffness; F, toughness) $(* \mathrm{p}<0.05, * * \mathrm{p}<0.01, * * * \mathrm{p}<0.001, * * * * \mathrm{p}<0.0001$, ANOVA followed by the

Dunnett's multiple comparison test). (G)-(J) A positional recruitment simulation, in which fiber 
70 interactions were steric and linear, reproduced experimentally-observed enthesis mechanics as a

71 function of abduction angle. In silico $(\mathrm{G})$ strength vs. displacement and (H) strength, stiffness,

72 toughness results normalized against the case when fibers were pulled uniaxially without the

73 geometric constraints. (I) The relationship between fiber engagement and displacement depended

74 on abduction angle, demonstrating that the energy absorbed in re-orienting and engaging fibers

75 drove the toughening behavior of the of attachment. (J) Enthesis architecture was optimized for

76 toughness: normalized toughness was generally higher than normalized strength through most

77 abduction angles.

78 
A

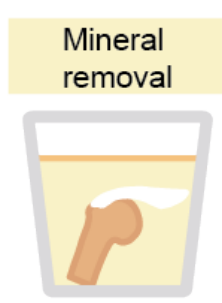

Formic Acid $72 \mathrm{~h}$

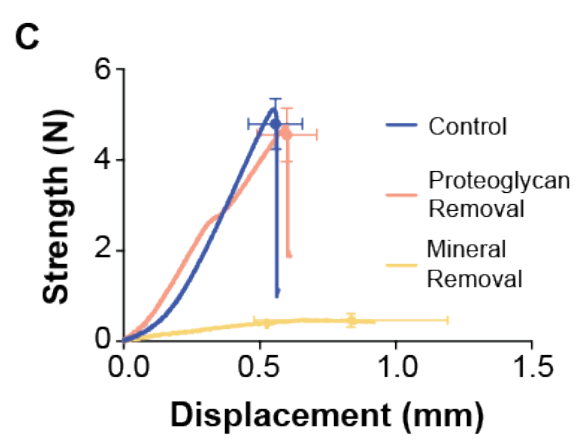

B
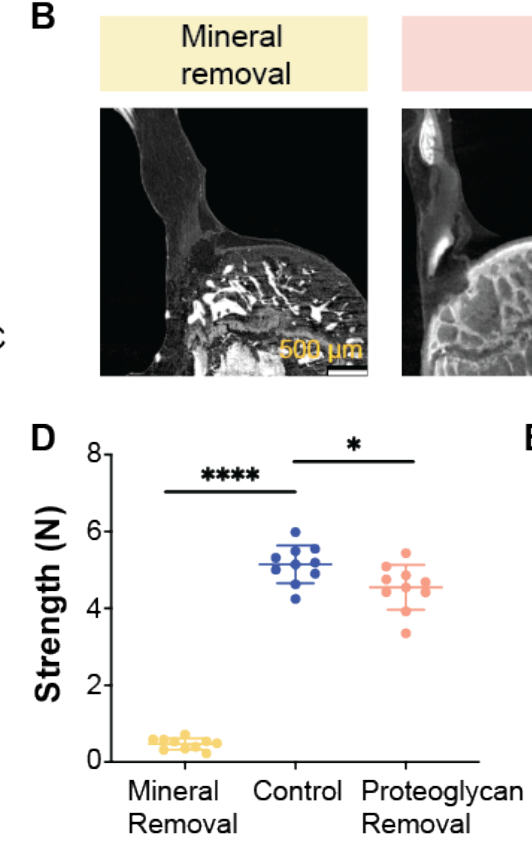

Proteoglycan removal
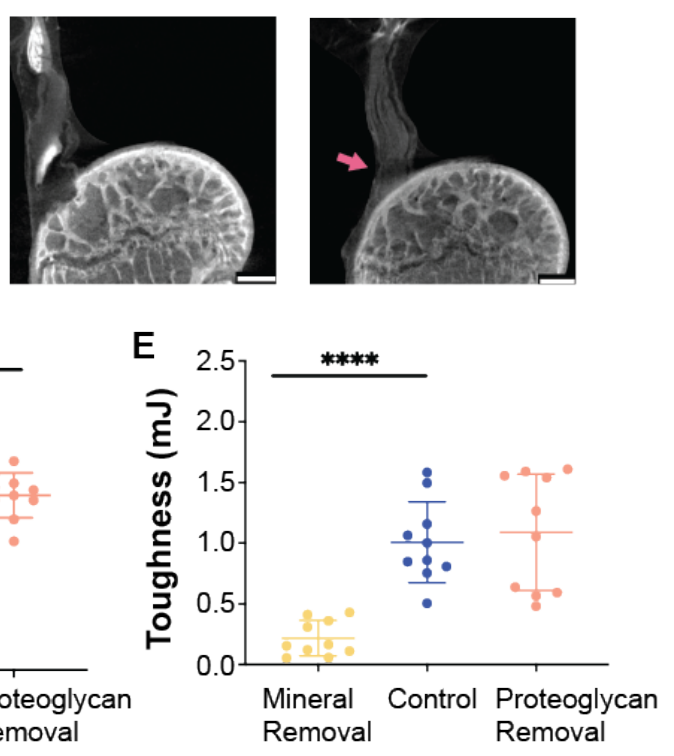

Fig. 4. Tendon enthesis composition drives enthesis mechanical properties.

(A) To examine compositional contributions to tendon-to-bone attachment strength and toughness, samples were immersed in decalcifying agent to completely remove mineral (A, left) or in Chondroitinase $\mathrm{ABC}$ for 5 days to chemically digest proteoglycans (A, right). (B) Postfailure contrast enhanced microCT scanning showed that loss of mineral or proteoglycan did not significantly alter the failure modes of the tendon enthesis. Most samples failed via bone avulsion, while a small number of samples depleted in proteoglycans failed at the edge of unmineralized fibrocartilage (pink arrow) (scale: $500 \mu \mathrm{m})$. (C)-(E), Quasi-static mechanical testing revealed significant differences in mechanical behavior of tendon entheses when mineral was removed. (C) Strength (failure force) vs. displacement behavior. (D) Removal of mineral led to a dramatic decrease in strength; removal of proteoglycan led to a relatively small decrease in strength. (E) Removal of mineral led to a significant decrease in toughness; removal of proteoglycan did not affect enthesis toughness. $(* \mathrm{p}<0.05, * * * * \mathrm{p}<0.0001$, ANOVA followed by the Dunnett's multiple comparison test). 
A

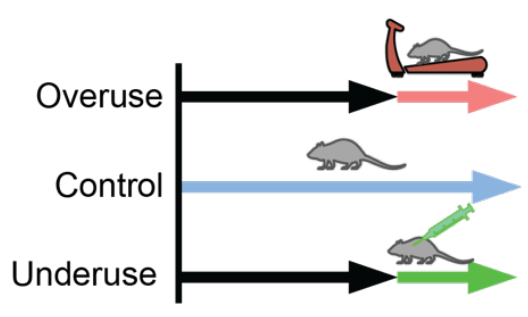

C

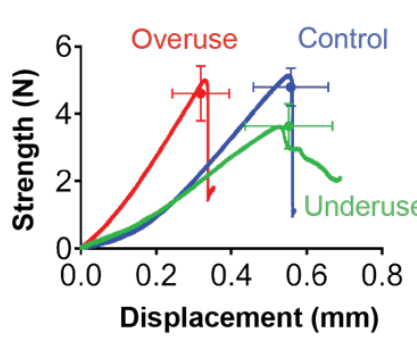

G

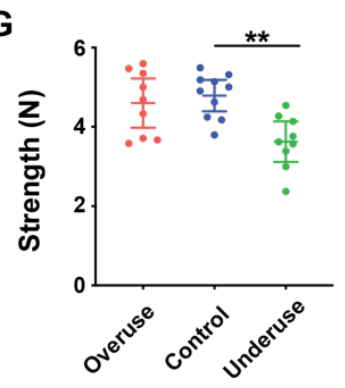

K
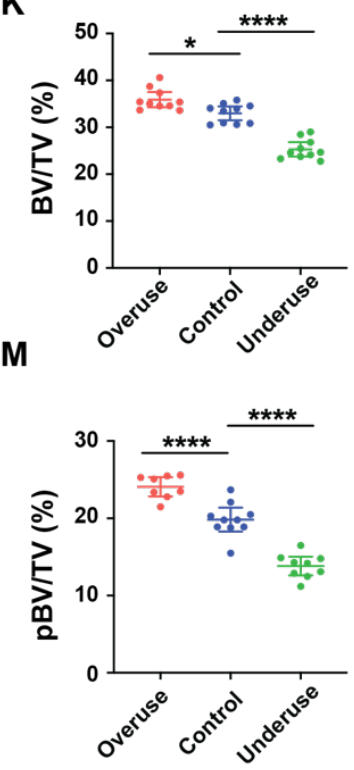

95

\section{composition.}

B

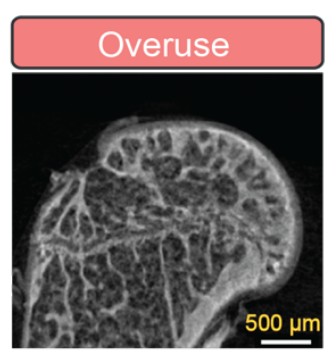

E

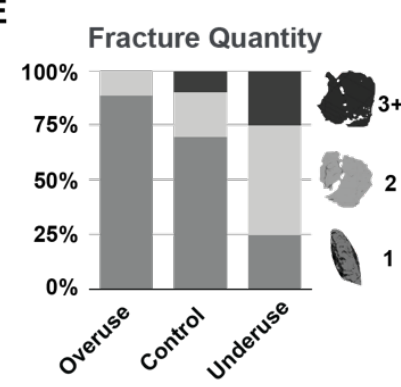

F
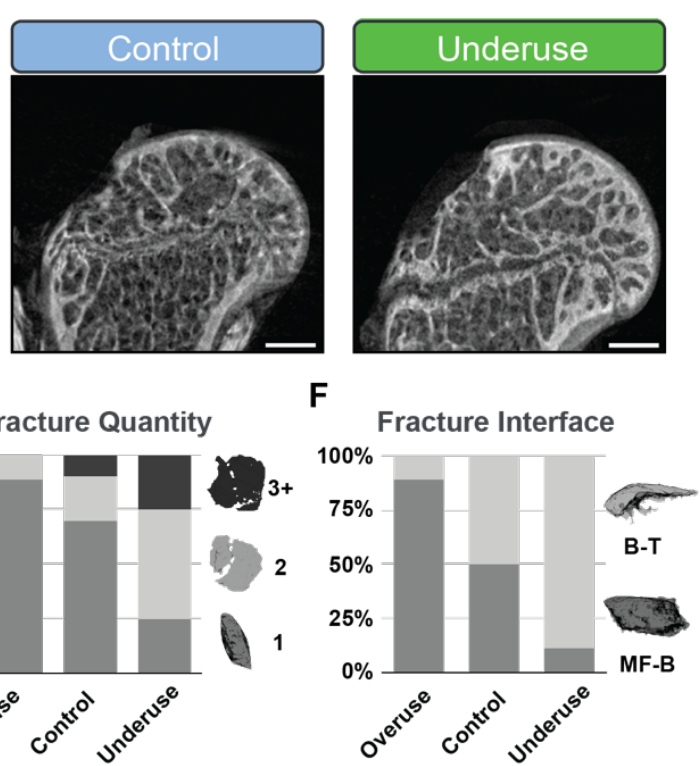

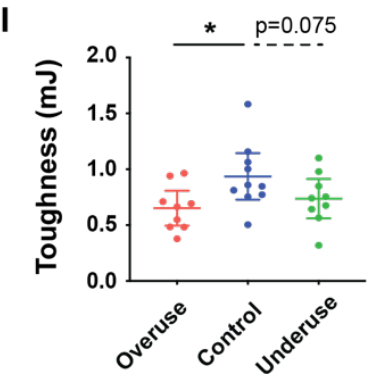

O

L

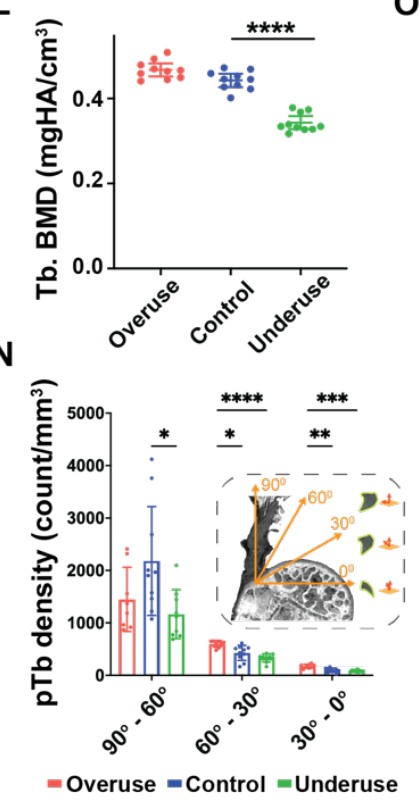

$\mathrm{H}$

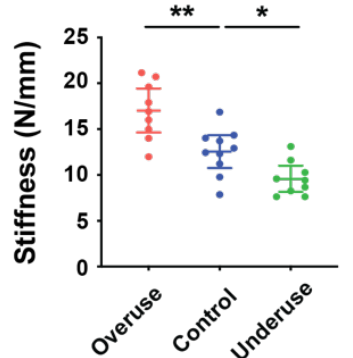

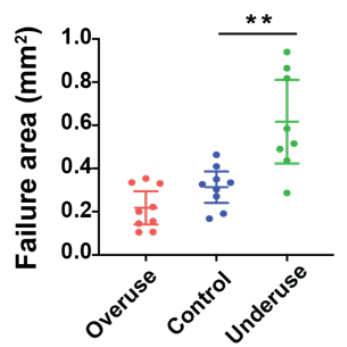

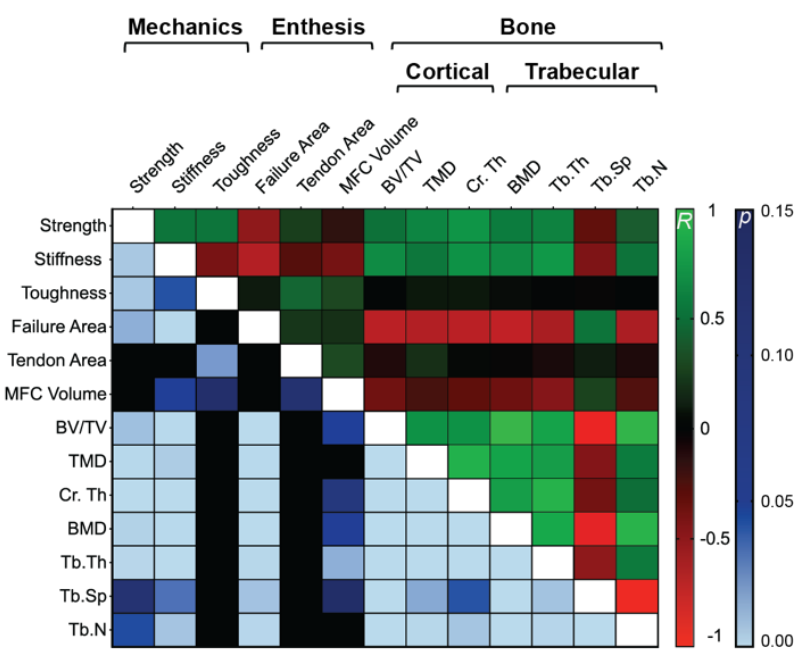

Fig. 5. The tendon enthesis actively adapts its architecture in vivo by modifying mineral

(A) 10-week-old mice were subjected to two degeneration models: underuse degeneration was induced via muscle paralysis and overuse degeneration was achieved through downhill treadmill 
jo running for 4 weeks. (B) Post-failure contrast enhanced microCT imaging revealed that

01 pathological entheses exhibited exclusively avulsion-type failures under tensile mechanical

02 testing (scale: $500 \mu \mathrm{m})$. (C)-(J), Physiological in vivo degeneration models reduced the ability of

03 the enthesis to protect against failure. (D) Failure area, (E) avulsed fragment quantity, and (F)

J4 failure interfaces were affected by enthesis pathology. Underuse degeneration led to $(\mathrm{G})$ lower

55 strength $(\mathrm{p}<0.01),(\mathrm{H})$ lower stiffness $(\mathrm{p}<0.05)$, and (I) trended with decreased toughness

J6 $(p=0.075)$ compared to that of control. Overuse degeneration decreased $(J)$ tendon cross-sectional

37 area $(\mathrm{p}<0.01),(\mathrm{H})$ stiffened the enthesis $(\mathrm{p}<0.01)$, and (I) significantly reduced toughness

08 compared to control $(\mathrm{p}<0.05)$. $(\mathbf{K})-(\mathbf{L})$, Bone morphometric analysis revealed that underuse led

99 to $(\mathrm{K})$ reduced bone volume $(\mathrm{BV} / \mathrm{TV})(\mathrm{p}<0.0001)$ and $(\mathrm{L})$ reduced bone mineral density $(\mathrm{BMD})$

10 in the bone underlying the attachment $(\mathrm{p}<0.0001)$. (M) The volume of load bearing trabecular

11 plates $(\mathrm{pBV} / \mathrm{TV})$ increased significantly $(\mathrm{p}<0.0001)$ due to overuse and decreased significantly

$12(\mathrm{p}<0.0001)$ due to underuse, with significant changes in their $(\mathbf{N})$ orientations $(\mathrm{p}<0.01,2$-way

13 ANOVA followed by Dunnet's multiple comparison test). (O) Enthesis strength correlated with

$14 \operatorname{BMD}(\mathrm{R}=0.60, \mathrm{p}<0.001)$, cortical thickness $(\mathrm{R}=0.69, \mathrm{p}<0.001)$, and trabecular plate thickness

$15(\mathrm{R}=0.44, \mathrm{p}<0.001)$. Enthesis toughness correlated with tendon cross-sectional area $(\mathrm{R}=0.44$,

$16 \mathrm{p}<0.01$, Pearson correlation). $(* \mathrm{p}<0.05, * * \mathrm{p}<0.01, * * * \mathrm{p}<0.001, * * * * \mathrm{p}<0.0001$, ANOVA

17 followed by the Dunnett's multiple comparison test unless otherwise reported). 


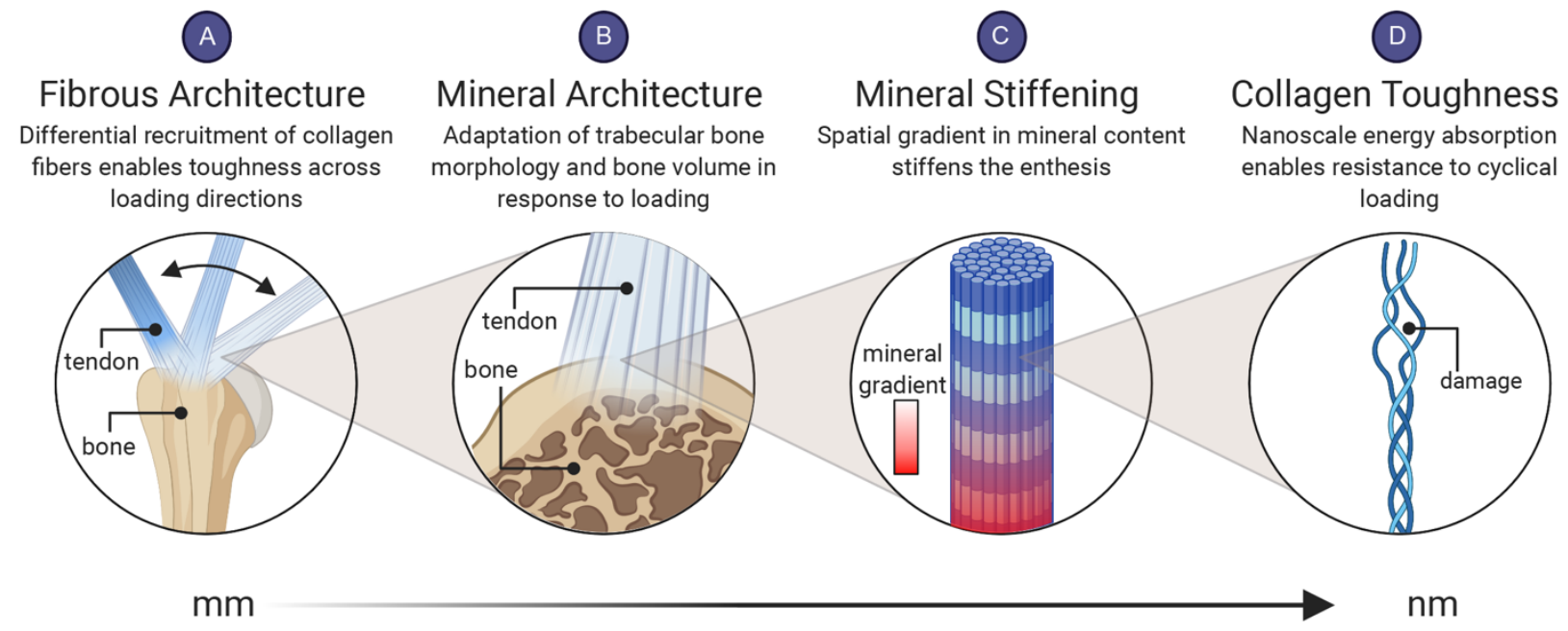

21 Fig. 6. The fibrous and mineral architectures of the tendon enthesis provide multiscale toughening mechanisms for a resilient attachment between tendon and bone.

23 Enthesis toughness is achieved over multiple length scales through unique fibrous and mineral architectures. At the millimeter length scale (A) the fibrous architecture of the tendon enthesis allows for fiber recruitment and re-orientation to optimize toughness over strength across a range of loading directions. At the micrometer length scale (B) the enthesis actively adapts its mineral

27 architecture to maintain its strength along the axis of loading. At the micrometer-to-nanometer 28 length scale (C) a spatial gradient in mineral across the enthesis reduced stress concentrations 29 (16). At the nanometer length scale (D) collagen damage localization protects against damage 30 prorogating to higher length scales. 